\title{
Suficiência amostral para alface cultivada em diferentes ambientes
}

\author{
Sample sufficiency for lettuce grown in different environments \\ Daniel Santos $^{\mathrm{I}}$ Fernando Machado Haesbaert $^{\mathrm{I}}$ Odair José Puhl $^{\mathrm{I}}$ \\ Jorge Roque Alves dos Santos ${ }^{I}$ Alessandro Dal'Col LúcioII
}

\section{RESUMO}

Com o objetivo de estimar o tamanho de amostra para alface cultivada em diferentes ambientes, foram conduzidos quatro cultivos com alface, dois deles foram realizados no ano de 2007, com a cultivar 'Vera', e dois foram realizados no ano de 2008, com a cultivar 'Amanda'. Os ambientes em 2007 foram estufa e a campo, e em 2008 foram estufa e túnel. Em ambos os anos de cultivo, foram avaliadas a fitomassa fresca e fitomassa seca. Para cada variável, em cada linha de cultivo, foram estimadas a média, a variância e o coeficiente de variação. Para testar a homogeneidade das variâncias entre as linhas, aplicou-se o teste de Bartlett, a 5\% de significância. Nos casos em que as variâncias das linhas foram homogêneas, calculou-se um CV\% médio para cada ambiente e este foi utilizado para o cálculo do tamanho de amostra. Nos casos em que as variâncias foram heterogêneas, utilizou-se o maior CV\% do ambiente para o cálculo do tamanho da amostra. Os resultados mostraram que, em $40 \%$ dos experimentos, quando a variável foi a fitomassa fresca, $e$ em $60 \%$, quando foi a seca, os diferentes ambientes de produção proporcionaram variabilidades significativamente diferentes entre as linhas de cultivo. Foi possível determinar que, com a semiamplitude do intervalo de confiança de $10 \%$, devem ser amostradas 30, 40 e 33 plantas na linha de cultivo, na estufa, no túnel e a campo respectivamente, para a fitomassa fresca das plantas. Já para a fitomassa seca das plantas devem ser amostradas 27, 29 e 32 plantas, respectivamente, para estufa, túnel e campo.

Palavras-chave: ambiente protegido, amostragem, Lactuca sativa L., precisão experimental.

\section{ABSTRACT}

The aim of this study was to estimate the sample size for lettuce cultivated in different environments, and for this

\begin{abstract}
four lettuce crops were cultivated. Two of them were conducted in 2007, with the cultivar 'Vera' and two in 2008 with the cultivar 'Amanda'. The environments in 2007 were greenhouse and field and in 2008, greenhouse and tunnel. In both years of cultivation the fresh biomass and dry weight were evaluated. For each variable in each row of plants was estimated the mean, variance and coefficient of variation (CV\%). To test the homogeneity of variances among the lines was applied Bartlett's test at 5\% significance level. In cases where the variances were homogeneous lines, was also calculated an average CV\% for each environment, and this $C V \%$ was used to calculate the sample size. In cases where variances were heterogeneous, the highest $C V \%$ of the environment for the calculation of sample size was used. The results showed that in $40 \%$ of trials when the variable was fresh biomass and $60 \%$ when it was dry, the different production environments provided variability significantly different between the rows of cultivation. It was found that, with the half-width confidence interval of $10 \% 30,40$ and 33 plants should be sampled in the row of plants in the greenhouse, tunnel and field respectively, for the fresh biomass of plants. Already for plants dry biomass, should be sampled 27, 29 and 32 plants, respectively, for greenhouse, tunnel and field.
\end{abstract}

Key words: experimental precision, Lactuca sativa L., Protected environment, sampling.

\section{INTRODUÇÃO}

A alface (Lactuca sativa L.) é uma cultura de grande importância econômica no Brasil. Atualmente ela é a hortaliça folhosa de maior consumo. Em 1996, sua produção foi de 311.888 toneladas (IBGE, 1996). Constitui uma importante fonte de sais minerais, principalmente de cálcio e de vitaminas, especialmente a vitamina $\mathrm{A}$.

'Curso de Agronomia, Centro de Ciências Rurais (CCR), Universidade Federal de Santa Maria (UFSM), 97105-900, Santa Maria, RS, Brasil.

"Departamento de Fitotecnia, CCR, UFSM, Santa Maria, RS, Brasil. E-mail: adlucio@smail.ufsm.br. Autor para correspondência. 
O Estado do Rio Grande do Sul (RS) apresenta períodos do ano com condições climáticas desfavoráveis ao desenvolvimento dessa cultura, pois, segundo BRUNINI et al. (1976), a faixa de temperatura para o crescimento da alface é de 7 a $24^{\circ} \mathrm{C}$ (média de $18^{\circ} \mathrm{C}$ ). Nos meses de inverno no RS, ocorrem frequentemente temperaturas inferiores a $10^{\circ} \mathrm{C}$ e ainda precipitações pluviométricas prolongadas, provocando danos físicos e retração do crescimento das plantas. Nos meses de verão, o problema ocorre em razão das temperaturas muito altas. Tendo em vista a redução dos efeitos negativos no período de inverno, uma das alternativas para viabilizar o cultivo da alface nessa estação é a proteção ambiental, seja ela com túnel, seja em estufas cobertas com filmes de polietileno.

A determinação do tamanho de parcela e de amostra, assim como do número de repetições, é uma importante estratégia no momento do planejamento experimental para reduzir o erro experimental, de acordo com STEEL et al. (1997) e STORCK et al. (2006). A amostragem é uma técnica amplamente utilizada no estudo de populações, decorrente das vantagens que esse processo proporciona, como o menor custo e a rapidez na obtenção e na análise dos dados, além de que os pesquisadores podem ser treinados e supervisionados de forma mais cuidadosa (BUNCHAFT \& KELLNER, 1998). FERNANDES \& SILVA (1996) salientam que, quanto maior for o tamanho da amostra, maior a precisão e, em consequência disso, o coeficiente de variação amostral tende a diminuir, pois um aumento no tamanho da amostra reduz a variância da média amostral, mas também maiores serão os gastos e o tempo para realização da amostragem. Por outro lado, amostras pequenas podem resultar em redução da precisão dos experimentos.

O tamanho da amostra, na experimentação agrícola, é um dos principais problemas a serem resolvidos pelos pesquisadores, conforme relatam STUKER \& BOFF (1998). Uma alternativa para solucionar esse problema é a determinação de uma amostra dimensionada para a precisão desejada (ESTEFANEL et al., 1984, ESTEFANEL et al., 1996b, SOUZA et al., 2002 e LÚCIO et al., 2003) e da homogeneidade dos elementos populacionais (CAMPOS, 1985). De acordo com SILVEIRA JÚNIOR et al. (1980), ao dimensionar uma amostra, necessita-se do conhecimento prévio da variância da população e do grau de precisão desejado, mas, quando não há informações sobre essa variabilidade, deve-se realizar uma pré-amostragem, em pequena escala, para que possam ser obtidas as estimativas dos parâmetros populacionais (média e variância) que serão usados na obtenção do melhor tamanho da amostra.
Trabalhos realizados para estimar o tamanho de amostra em experimentos realizados em ambientes protegidos, onde a área é limitada, mostram que existe variabilidade significativa entre as linhas de cultivo, independente da cultura avaliada (SOUZA et al., 2002 e LÚCIO et al., 2003). Já para a cultura da alface MARODIM et al. (2000) apresentaram como tamanho de amostra para fitomassa fresca de alface hidropônica o valor de 40 plantas para uma semiamplitude do intervalo de confiança (D\%) de 5\% e de sete plantas para $\mathrm{D} \%=20$. Também trabalhando com alface, SANTOS et al. (2007) recomendam que devam ser amostradas três plantas por perfil hidropônico, com frequência de três em três dias, para ajustes de curvas de crescimento, considerando a fitomassa seca total da planta.

Diante disso, é importante estimar um tamanho de amostra para cada situação de cultivo, evitando trabalhar com amostras super ou subdimensionadas. O objetivo do trabalho foi estimar o tamanho de amostra para experimentos com alface conduzidos em estufa, em túnel e a campo.

\section{MATERIAL E MÉTODOS}

Foram conduzidos quatro cultivos com alface na área experimental do Departamento de Fitotecnia (latitude 29 43'28.23”S, longitude $53^{\circ} 43^{\prime} 17.22^{\prime}$ 'O e altitude $104 \mathrm{~m}$ ), no Campus da Universidade Federal de Santa Maria, no Município de Santa Maria, RS. O clima da região, segundo a classificação de Köeppen (MORENO, 1961), é do tipo Cfa - temperado chuvoso, com chuvas bem distribuídas ao longo do ano e subtropical do ponto de vista térmico. O solo é classificado, no Sistema Brasileiro de Classificação de Solos (EMBRAPA, 1999), como Argissolo Vermelho Distrófico arênico.

Dois experimentos em branco foram realizados no ano de 2007, com a cultivar 'Vera', e dois foram realizados no ano de 2008, com a cultivar 'Amanda'. Para cada cultivar, foram realizados dois cultivos em épocas diferentes, sendo o primeiro de $02 /$ 01/07 a 30/01/07 (verão) e o segundo de 16/06/07 a 19/ 07/07 (inverno). No ano de 2008, o primeiro cultivo foi de 03/04/08 a 16/05/08 (outono), e o segundo foi de 10/ 10/08 a 22/11/08 (primavera). As mudas utilizadas foram cultivadas em bandejas de 288 alvéolos, com o uso do substrato HORTMIX. O transplante foi realizado quando as mudas estavam com 30 dias contados da emergência, apresentando três folhas distintas.

Os experimentos realizados no ano de 2007 foram conduzidos em uma estufa plástica, com estrutura metálica do tipo arco pampeano, pé direito de $2 \mathrm{~m}$ e $3,5 \mathrm{~m}$ na parte central, com $20 \mathrm{~m}$ de comprimento e 
orientada no sentido norte-sul, e também a campo. Já os experimentos realizados no ano de 2008 foram conduzidos em dois tipos de ambientes protegidos, uma estufa e dois túneis. Os túneis plásticos são idênticos, ambos possuindo pé direito de $3 \mathrm{~m}, 20 \mathrm{~m}$ de comprimento e orientação norte-sul, enquanto que a estufa foi a mesma utilizada em 2007. Nos ambientes protegidos dos dois anos de cultivo, a cobertura foi feita com filme de polietileno de baixa densidade (PEBD), com espessura de 100 micras e aditivo antiUV.

Em todos os experimentos, as plantas foram dispostas em linhas (camalhões sem o uso de mulching) espaçadas de um metro, e o espaçamento entre plantas foi de $0,3 \mathrm{~m}$. No primeiro ano de cultivo na estufa e a campo, foram dispostas linhas contendo 48 plantas cada. Na estufa, foram oito linhas nas duas estações sazonais e no campo foram seis linhas no verão e três linhas no inverno. Já no segundo ano de cultivo, nas duas estações, na estufa, foram utilizadas oito linhas com 50 plantas cada e em ambos os túneis três linhas com 60 plantas cada. Todos os tratos culturais foram aplicados de acordo com a recomendação da cultura.

$\mathrm{Na}$ estufa, em todos os experimentos, foram consideradas somente as seis linhas centrais para fins de análise, e nos demais ambientes todas as linhas foram consideras. Para determinar as fitomassas fresca (FF) e seca da parte aérea (FS), foram colhidas, no período da manhã, as partes aéreas de todas as plantas das linhas consideradas. Estas foram acondicionadas em sacos de papel e pesadas em balança digital com precisão de $1 \mathrm{~g}$, para determinação da FF. Após a primeira pesagem, as partes aéreas foram levadas para estufa de circulação de ar forçado à temperatura de 65 $70^{\circ} \mathrm{C}$, por 48 horas, quando atingiram massa constante, para, em seguida, proceder à segunda pesagem para a determinação da FS por planta.

Para cada variável em cada linha de cultivo, foram estimados a média $(\overline{\mathrm{X}})$, a variância $\left(\mathrm{s}^{2}\right)$ e o coeficiente de variação (CV\%). Para testar a homogeneidade das variâncias entre as linhas de cultivos e verificar se estas poderiam ser representadas por uma mesma variância, aplicou-se o teste de Bartlett (STEEL et al., 1997), a 5\% de probabilidade. Nos casos em que as variâncias das linhas foram homogêneas, calculou-se também um $\mathrm{CV} \%$ médio para cada ambiente. Quando as variâncias das linhas foram homogêneas, utilizou-se, para o cálculo do tamanho de amostra, o CV\% médio do ambiente. Nos casos em que as variâncias foram heterogêneas, utilizou-se o maior $\mathrm{CV} \%$ do ambiente. Também foram realizados testes F, a 5\% de probabilidade, para comparar as variâncias entre os ambientes de cultivo, dentro de cada variável e cada ano de experimentação.

$$
\text { Pela expressão }
$$

(COCHRAN,

1977), estimou-se o tamanho ideal de amostra para cada linha de cultivo, na qual $n$ é o tamanho de amostra para população infinita, $\mathrm{t}_{\alpha / 2}$ é o valor da tabela $\mathrm{t}$ de Student com n-1 graus de liberdade a $5 \%$ de probabilidade, $\mathrm{CV} \%$ é o coeficiente de variação das fitomassas das plantas e D\% é a semiamplitude do intervalo de confiança da média $(\mathrm{D} \%=5,10,15$ e 20$)$.

Como a população é finita, houve a necessidade de realizar a correção para obter o tamanho final da amostra. Para tanto, foi seguida a recomendação

deCOCHRAN (1977), aplicando-se a fórmula $\mathrm{nc}=\frac{\mathrm{n}}{1+\frac{\mathrm{n}}{\mathrm{N}}}$, em que nc é o tamanho corrigido da amostra, $\mathrm{N}$ é o tamanho da população de cada linha de cultivo, e n é o tamanho da amostra para população infinita.

\section{RESULTADOS E DISCUSSÃO}

Os resultados do teste de homogeneidade das variâncias entre as linhas de cultivo, dentro de cada experimento, mostraram que, em cultivos com a cultivar 'Vera', a homogeneidade foi evidente em 75\% dos experimentos para a fitomassa fresca (FF), enquanto que para a fitomassa seca (FS) em nenhum dos casos ocorreu essa homogeneidade (Tabela 1). Já para cultivos com a cultivar 'Amanda' a homogeneidade entre as variâncias foi verificada em 50 e $67 \%$ dos experimentos, respectivamente, para a FF e FS. Esses resultados mostram que os diferentes ambientes de produção proporcionam variabilidades significativamente diferentes entre as linhas de cultivo, sendo também influenciadas pela estação sazonal de cultivo e cultivar utilizada, concordando com trabalhos de LÚCIO et al. (2008) e CARPES et al. (2008).

Para os cultivos de 2007 na estufa, tanto no verão, como no inverno, as variâncias foram significativamente maiores que a campo, para as duas variáveis, exceto para FS no inverno, em que as variabilidades dos ambientes não diferiram significativamente, pelo teste F (Tabela 1). Porém, nos casos a campo, foram obtidos maiores valores de $\mathrm{CV}$ (Tabela 1)e, como consequência, maiores foram também os tamanhos de amostra (Tabela 2). No verão, as variâncias foram significativamente maiores do que no inverno nas duas variáveis, tanto a campo, como na estufa. Os CVs também foram maiores a campo para a FF na estufa independente da estação do ano (Tabela 1). Isso mostra que as condições de verão proporcionam um grau de variabilidade maior aos 
Tabela 1 - Variâncias por linha de cultivo e do experimento e coeficiente de variação máximo (CV\%) por linha de cultivo, para as fitomassas fresca (FF) e seca (FS), em gramas, de duas cultivares de alface em diferentes ambientes de produção. Santa Maria - RS, 2009.

\begin{tabular}{|c|c|c|c|c|c|c|c|c|c|c|c|c|}
\hline \multicolumn{13}{|c|}{ 'Vera' } \\
\hline & \multicolumn{6}{|c|}{-------------------------Verão/2007-------------------------- } & \multicolumn{6}{|c|}{ 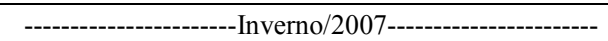 } \\
\hline & \multicolumn{3}{|c|}{----------Estufa------------ } & \multicolumn{3}{|c|}{----------Campo---------- } & \multicolumn{3}{|c|}{----------Estufa---------- } & \multicolumn{3}{|c|}{----------Campo---------- } \\
\hline & $\mathrm{FF}$ & & $\mathrm{FS}^{1}$ & FF & & $\mathrm{FS}^{1}$ & $\mathrm{FF}$ & & $\mathrm{FS}^{1}$ & $\mathrm{FF}^{1}$ & & $\mathrm{FS}^{1}$ \\
\hline Linha 1 & 6374 & & 14,0 & 2538 & & 7,2 & 1656 & & 1,6 & 347 & & 1,6 \\
\hline Linha 2 & 5929 & & 9,6 & 3304 & & 6,3 & 1648 & & 5,1 & 747 & & 4,1 \\
\hline Linha 3 & 1101 & & 32,2 & 3204 & & 11,9 & 1876 & & 3,8 & 594 & & 3,1 \\
\hline Linha 4 & 6293 & & 23,2 & 4131 & & 8,1 & 1565 & & 1,8 & - & & - \\
\hline Linha 5 & 6155 & & 19,1 & 3471 & & 11,5 & 1784 & & 3,4 & - & & - \\
\hline Linha 6 & 6615 & & 8,8 & 3363 & & 17,6 & 1564 & & 4,9 & - & & - \\
\hline Experimento & 7063 & & $32,2^{2}$ & 3335 & & $17,6^{2}$ & 1682 & & $4,9^{2}$ & $747^{2}$ & & $4,1^{2}$ \\
\hline \multirow[t]{4}{*}{$\mathrm{CV} \%$} & 35,7 & & $39,3^{3}$ & 52,3 & & $50,4^{3}$ & 34,0 & & $25,4^{3}$ & 41,7 & & $27,2^{3}$ \\
\hline & \multirow{2}{*}{\multicolumn{2}{|c|}{-------------------'--Estufa----- }} & \multicolumn{4}{|c|}{ 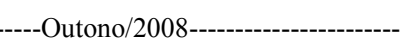 } & \multicolumn{6}{|c|}{----------------------Primavera/2008---------------------- } \\
\hline & & & \multicolumn{2}{|c|}{-----Túnel 1----- } & \multicolumn{2}{|c|}{-----Túnel 2----- } & \multicolumn{2}{|c|}{-----Estufa----- } & \multicolumn{2}{|c|}{-----Túnel 1----- } & \multicolumn{2}{|c|}{-----Túnel 2----. } \\
\hline & $\mathrm{FF}^{1}$ & FS & $\mathrm{FF}^{1}$ & FS & FF & FS & $\mathrm{FF}^{1}$ & $\mathrm{FS}^{1}$ & $\mathrm{FF}$ & $\mathrm{FS}^{1}$ & FF & FS \\
\hline Linha 1 & 6408 & 11,9 & 21606 & 10,8 & 9044 & 32,8 & 7429 & 17,4 & 61445 & 148,4 & 16651 & 33,1 \\
\hline Linha 2 & 8475 & 12,8 & 3245 & 7,4 & 11347 & 23,5 & 7251 & 21,2 & 46626 & 87,8 & 22932 & 52,3 \\
\hline Linha 3 & 5577 & 11,7 & 5668 & 11,4 & 10989 & 21,7 & 16300 & 39,5 & 35709 & 64,6 & 24984 & 53,9 \\
\hline Linha 4 & 6909 & 15,5 & - & - & - & - & 15170 & 34,8 & - & - & - & - \\
\hline Linha 5 & 3244 & 8,3 & - & - & - & - & 9554 & 29,3 & - & - & - & - \\
\hline Linha 6 & 2996 & 6,5 & - & - & - & - & 7758 & 19,8 & - & - & - & - \\
\hline Experimento & $8475^{2}$ & 11,1 & $21606^{2}$ & 9,9 & 10460 & 26,0 & $7758^{2}$ & $39,5^{2}$ & 47927 & $148,4^{2}$ & 21522 & 46,4 \\
\hline $\mathrm{CV} \%$ & $43,0^{3}$ & 33,3 & $71,7^{3}$ & 30,9 & 34,3 & 32,8 & $32,1^{3}$ & $28,2^{3}$ & 40,8 & $40,9^{3}$ & 22,0 & 19,2 \\
\hline
\end{tabular}

${ }^{1}$ variâncias heterogêneas entre linhas de cultivo pelo teste de Bartlett, a 5\% de probabilidade.

${ }^{2}$ variância da linha de maior $\mathrm{CV} \%$.

${ }^{3} \mathrm{CV} \%$ máximo.

cultivos, havendo necessidade de um maior tamanho de amostra (Tabela 2).

Em 2008, os dois túneis apresentaram variâncias estatisticamente superiores às da estufa, pelo teste F, comparando variâncias duas a duas (Tabela 1). Porém, no experimento em estufa, em razão das maiores razões ocorridas entre desvio padrão e média, que geraram um maior $\mathrm{CV}$, os tamanhos de amostra foram maiores que no túnel 2 no outono e na primavera, para a FF, e na primavera, para FS (Tabela 2). O túnel 1 apresentou maiores variâncias e também maiores tamanhos de amostra que o túnel 2, exceto para FS no outono, em que a variabilidade foi maior no túnel 2 (Tabela 1), e os tamanhos de amostra se equivaleram (Tabela 2). Nos ambientes estudados para a cultivar 'Amanda', para ambas variáveis, excluindo-se a FF na estufa em que as variâncias não diferiram, foram obtidas variabilidades significativamente maiores na primavera. No entanto, os valores de CV\% foram menores nessa estação em razão das médias relativamente altas, com exceção da FS no túnel 1, em que o valor foi maior na primavera (Tabela 1).
No ambiente estufa plástica, quando avaliada a FF da cultivar 'Vera', foi observado um tamanho de amostra similar nas duas estações sazonais, mostrando que a alteração da estação, apesar de ter alterado significativamente as variâncias, teve pouca influência sobre o coeficiente de variação desse ambiente. Já para o cultivo a campo, a mudança da estação de cultivo fez com que o tamanho de amostra se alterasse em maior proporção (Tabela 2). Houve uma redução desse tamanho no inverno, evidenciando que a significativa alteração na variância ocorrida foi acompanhada de uma maior modificação do CV (Tabela 1). Essa condição mostra que, ao se trabalhar em ambiente controlado, como o caso da estufa plástica, há uma manutenção no CV do ambiente, em razão da formação de um microclima dentro do sistema, mantendo assim o comportamento da produção das plantas, principalmente nos meses de inverno, fato não observado em cultivos a campo, ficando mais vulneráveis às modificações de clima.

Para a mesma cultivar 'Vera', no verão, ao se avaliar a FS, as estimativas do tamanho de amostra 
Tabela 2 - Tamanho de amostra em numero de plantas por linha para as fitomassas fresca (g) e seca (g) de duas cultivares de alface em diferentes ambientes de produção, para diferentes semiamplitudes do intervalo de confiança (D\%). Santa Maria - RS, 2009.

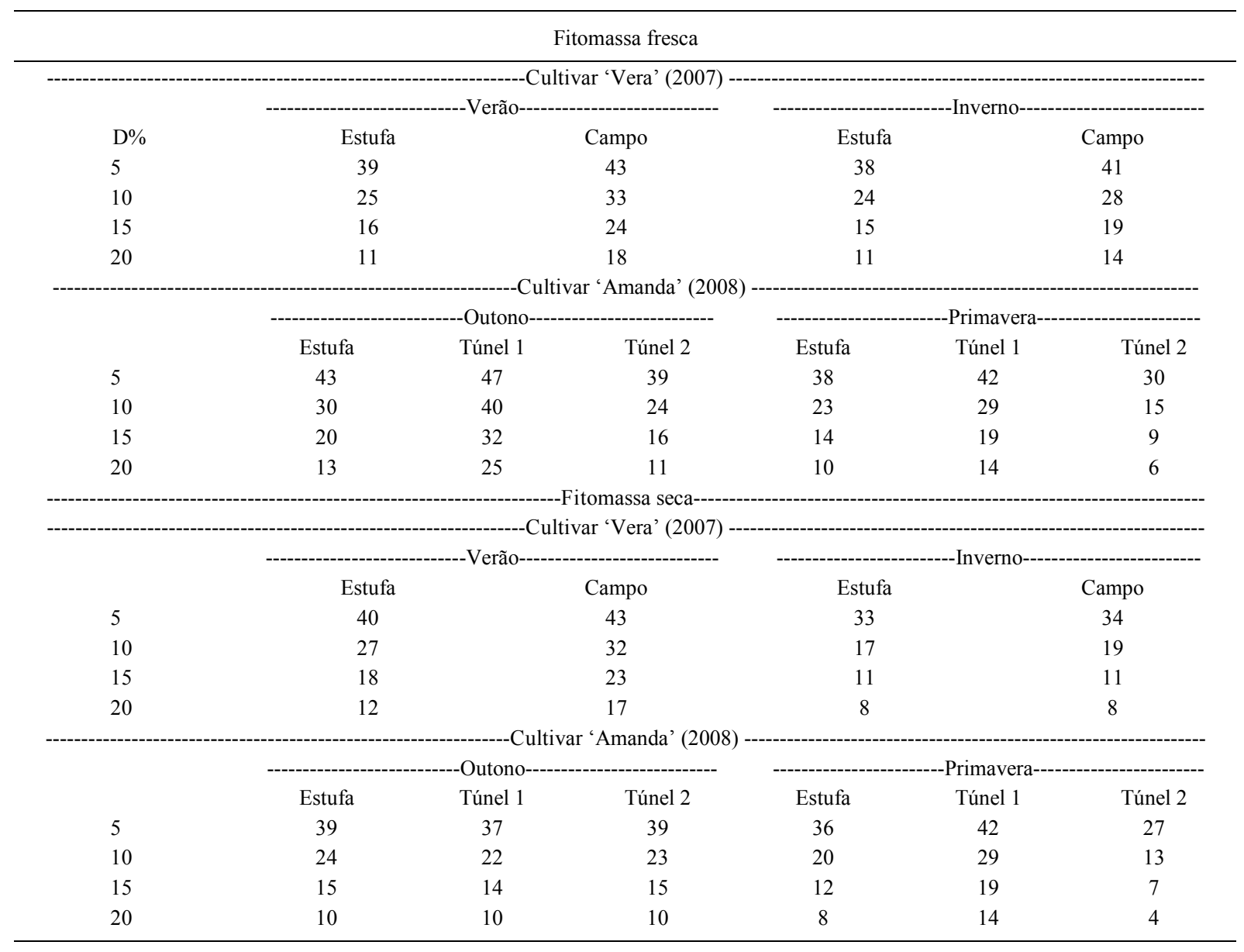

foram próximas àquelas observadas para a FF. Já no cultivo de inverno as estimativas para a FS foram menores, quando comparadas com a FF, mas o comportamento de maiores amostras para o cultivo a campo se manteve.

Nos cultivos realizados em 2008, com a cultivar 'Amanda', os tamanhos de amostra para a FF foram menores na primavera, tanto para a estufa, quanto para os túneis, em virtude das menores razões obtidas entre desvio padrão e média geral. No entanto, para a FS, nem todos os ambientes mantiveram esse comportamento, pois o maior tamanho de amostra para o túnel 1 ocorreu na primavera, enquanto que para túnel 2 e estufa foi no outono.

Foi possível perceber que, quando a variável utilizada foi a FS, os tamanhos de amostra foram menores do que quando foi a FF, com exceção da estufa em 2007, no verão, que apresentou um maior tamanho de amostra para a FS e do túnel 1 na primavera de 2008, do campo no verão de 2007 e do túnel 2 no outono
2008, em que os tamanhos se equivaleram. Isso mostra que, na maioria dos casos, a secagem das plantas faz com que haja uma diminuição da variabilidade.

Os resultados mostram que, para a estufa, adotando-se o $\mathrm{D} \%$ de $10 \%$, independentemente da estação do ano que se cultiva, da cultivar utilizada e da variável ser FS ou FF, é possível utilizar um tamanho de amostra equivalente a $60 \%$ da população, sem que ele seja subestimado. Esse resultado evidencia que $40 \%$ das plantas podem deixar de ser colhidas, ou seja, é possível se obter uma economia de $40 \%$ em mão-deobra, em tempo para colheita e pesagem e em sacos para acondicionar as plantas para secagem. Para o ambiente campo, no $\mathrm{D} \%=10$, para a cultivar 'Vera', adotando-se um tamanho de amostra de $69 \%$ da população, ele não é subestimado, independente da variável analisada ou da época de cultivo. Com isso, as economias seriam da ordem de $31 \%$. Para os túneis, mantendo $\mathrm{D} \%=10$, quando utilizada a cultivar 'Amanda', amostrar $67 \%$ da população, tanto para

Ciência Rural, v.40, n.4, abr, 2010. 
cultivos do outono, quanto para cultivos da primavera, independente da variável, é suficiente para manter o tamanho da amostra com um tamanho adequado. Nesse caso, é possível uma economia de 33\%.

\section{CONCLUSÕES}

O tamanho de amostra para alface varia em função do ambiente, da época de cultivo, da cultivar utilizada e da variável observada.

Para uma única recomendação de amostragem, com a semiamplitude do intervalo de confiança (D\%) de 10\%, é possível amostrar 30, 40 e 33 plantas na linha de cultivo para estufa, túnel e campo, respectivamente, para a fitomassa fresca das plantas. Já para a fitomassa seca das plantas é possível amostrar, na linha, 27, 29 e 32 plantas, respectivamente, para estufa, túnel e campo.

\section{AGRADECIMENTOS}

Ao Conselho Nacional de Desenvolvimento Científico e Tecnológico $(\mathrm{CNPq})$ e à Fundação de Apoio à Pesquisa do Estado do Rio Grande do Sul (FAPERGS), pelo auxílio financeiro e pelas bolsas de iniciação científica e de produtividade em pesquisa.

\section{REFERÊNCIAS}

BRUNINI, O. et al. Temperatura-base para alface cultivar 'white boston', em um sistema de unidades térmicas. Bragantia, Campinas, v.35, n.1, p. 213-219, 1976.

BUNCHAFT, G.; KELNER, S.R.O. Estatística sem mistérios Petrópolis: Vozes, 1998. V.2, $221 \mathrm{p}$.

CAMPOS, H. Estatística e experimentação agronômica amostragem I. Piracicaba: ESALQ - USP, 1985. 17p.

CARPES, R. H. et al. Ausência de frutos colhidos e suas interferências na variabilidade da fitomassa de frutos de abobrinha italiana cultivada em diferentes sistemas de irrigação. Revista CERES, Viçosa, v.55, n.6, p.590-595, 2008. Disponível em: $<$ h t t p : / / w w w. ceres.ufv.br/CERES/revistas/ V55N006P37108.pdf>. Acesso em: 27 mar. 2009.

COCHRAN, W.G. Técnicas de amostragem. 2.ed. Rio de Janeiro: Fundo de Cultura, 1977. 555p.

EMBRAPA. Centro Nacional de Pesquisa de Solos (Rio de Janeiro, RJ). Sistema brasileiro de classificação dos solos. Brasília: Embrapa-SPI, 1999. 412p.

ESTEFANEL, V. et al. Tamanho da amostra para estimar características agronômicas da soja. Revista do Centro de Ciências Rurais, Santa Maria, v.14, n.3-4, p.221-229, 1984.
Tamanho da amostra para avaliação de componentes do rendimento na cultura do feijoeiro. Ciência Rural, Santa Maria, v.26, n.3, p.367-370, 1996. Disponível em: <http:// w w w. s c i e lo.b r / s c i e 1 o.p h p ? p i d = S $0103-$ $84781996000300004 \&$ script $=$ sci $\operatorname{arttext} \& \operatorname{tn} \mathrm{l}=\mathrm{pt}>$. Acesso em: 14 mar. 2009 . doi: $10.1590 / \bar{S} 0103-84781996000300004$.

FERNANDES, E.N.; SILVA, P.S.L. Tamanho da amostra e método de amostragem para caracteres da espiga do milho. Ciência e Agrotecnologia, Lavras, v.20, n.2, p.252-256, 1996.

INSTITUTO BRASILEIRO DE GEOGRAFIA E ESTATÍSTICA. Censo agropecuário: Brasil. Rio de Janeiro, 1996. Acessado em 20 ago. 2009. Online. Disponível em: h t t p ://w w w. sidra.ibge.gov.br/b d a/tabela/ protabl.asp?c $=524 \& \mathrm{z}=\mathrm{t} \& \mathrm{o}=1 \& \mathrm{i}=\mathrm{P}$.

LÚCIO, A.D. et al. Tamanho da amostra e método de amostragem para avaliação de características do pimentão em estufa plástica. Horticultura Brasileira, Brasília, v.21, n.2, p.180-184, 2003. Disponível em: <http://www.scielo.br/ s c i e lo.php? s cript=sci arttext\&pid=S 0102 $05362003000200012 \& \operatorname{lng}=\mathrm{en} \& \mathrm{nrm}=\mathrm{iso} \& \mathrm{t} \operatorname{lng}=\mathrm{pt}>$. Acesso em: 14 mar. 2009. doi: 10.1590/S0102-05362003000200012.

LÚCIO, A.D. et al. Variância e média da massa de frutos de abobrinha-italiana em múltiplas colheitas. Horticultura Brasileira, Brasília, v.26, n.3, p.335-341, 2008. Disponível em: $<$ http://www.scielo.br/scielo.php? pid=S 0102 $05362008000300009 \&$ script $=$ sci_arttext\&tlng $=\mathrm{g}>$. Acesso em: 14 mar. 2009. doi: 10.1590/S0102-05362008000300009.

MARODIM, V.S. et al. Delineamento experimental e tamanho de amostra para alface cultivada em hidroponia. Ciência Rural, Santa Maria, v.30, n.5, p.779-781, 2000. Disponível em: <http:/ /www.scielo.br/scielo.php?script $=$ sci arttext\&pid $=\mathrm{S} 0103$ $84782000000500006 \& \operatorname{lng}=\mathrm{pt} \& \mathrm{nrm}=\mathrm{iso} \& \mathrm{t} \operatorname{lng}=\mathrm{pt}>$. Acesso em: 14 mar. 2009. doi: 10.1590/S0103-84782000000500006.

MORENO, J.A. Clima no Rio Grande do Sul. Porto Alegre: Secretaria da Agricultura, 1961. 41p.

SANTOS, P.M. et al. Cronograma de amostragem de plantas de alface hidropônica para ajuste de curvas de crescimento. Ciência Rural, Santa Maria, v.37, n.6, p.1601-1608, 2007. Disponível em: <http:/ /www.scielo.br/scielo.php? script $=$ sci arttext\&pid $=S 0100$ $54052008000100021 \& \operatorname{lng}=\mathrm{en} \& \mathrm{nrm}=\mathrm{iso} \& \mathrm{t} \operatorname{lng}=\mathrm{pt}>$. Acesso em: 26 mar. 2009. doi: 10.1590/S0103-84782007000600015.

SILVEIRA JÚNIOR, P. et al. Estatística geral - inferência estatística. Pelotas: UFPEL, 1980. V.4, 156p.

SOUZA, M.F. et al. Tamanho da amostra para peso da massa de frutos, na cultura da abóbora italiana em estufa plástica. Revista Brasileira de Agrociência, Pelotas, v.8, n.2, p.123-128, 2002. Disponível em: < http://www.ufpel.tche.br/faem/agrociencia/ v8n2/artigo07.pdf>. Acesso em: 14 mar. 2009.

STEEL, R.G.D. et al. Principles and procedures of statistics biometrical approach. 3.ed. Nova York: McGraw-Hill, 1997. $666 \mathrm{p}$.

STORCK, L. et al. Experimentação vegetal. 2.ed. Santa Maria: UFSM, 2006. 198p.

STUKER, H.; BOFF, P. Tamanho da amostra na avaliação da queima-acinzentada em canteiros de cebola. Horticultura Brasileira, Brasília, v.16, n.1, p.10-13, 1998. 